\title{
Respiratory viral sepsis: epidemiology, pathophysiology, diagnosis and treatment
}

\author{
Xiaoying $\mathrm{Gu}^{1,2,3}$, Fei Zhou ${ }^{1,2}$, Yeming Wang ${ }^{1,2}$, Guohui Fan ${ }^{1,2,3}$ and Bin Cao $1,2,4,5$ \\ Affiliations: ${ }^{1}$ Dept of Pulmonary and Critical Care Medicine, National Clinical Research Center of Respiratory \\ Diseases, China-Japan Friendship Hospital, Beijing, China. ${ }^{2}$ Institute of Respiratory Medicine, Chinese \\ Academy of Medical Science, Beijing, China. ${ }^{3}$ Institute of Clinical Medical Sciences, China-Japan Friendship \\ Hospital, Beijing, China. ${ }^{4}$ Dept of Respiratory Medicine, Capital Medical University, Beijing, China. ${ }^{5}$ Tsinghua \\ University-Peking University Joint Center for Life Sciences, Beijing, China.
}

Correspondence: Bin Cao, Dept of Pulmonary and Critical Care Medicine, National Clinical Research Center of Respiratory Diseases, China-Japan Friendship Hospital, 2 East Yinghua Street, Beijing, 100029, China. E-mail: caobin_bena163.com

@ERSpublications

The disease burden of sepsis from viruses is great. Although current understanding of sepsis has improved, the differences between viral and bacterial sepsis were not well understood. It is urgent to pay more attention to respiratory viral sepsis. https://bit.ly/2Vujpfl

Cite this article as: Gu X, Zhou F, Wang Y, et al. Respiratory viral sepsis: epidemiology, pathophysiology, diagnosis and treatment. Eur Respir Rev 2020; 26: 200038 [https://doi.org/10.1183/16000617.0038-2020].

ABSTRACT According to the Third International Consensus Definition for Sepsis and Septic Shock, sepsis is a life-threatening organ dysfunction resulting from dysregulated host responses to infection. Epidemiological data about sepsis from the 2017 Global Burden of Diseases, Injuries and Risk Factor Study showed that the global burden of sepsis was greater than previously estimated. Bacteria have been shown to be the predominant pathogen of sepsis among patients with pathogens detected, while sepsis caused by viruses is underdiagnosed worldwide. The coronavirus disease that emerged in 2019 in China and now in many other countries has brought viral sepsis back into the vision of physicians and researchers worldwide. Although the current understanding of the pathophysiology of sepsis has improved, the differences between viral and bacterial sepsis at the level of pathophysiology are not well understood. Diagnosis methods that can broadly differentiate between bacterial and viral sepsis at the initial stage after the development of sepsis are limited. New treatments that can be applied at clinics for sepsis are scarce and this situation is not consistent with the growing understanding of pathophysiology. This review aims to give a brief summary of current knowledge of the epidemiology, pathophysiology, diagnosis and treatment of viral sepsis.

\section{Introduction}

Sepsis is a complex syndrome that results from infection. Recognising sepsis as not just an inflammatory disorder was one of the key reasons to revise previous criteria of sepsis, which had the limitation of low specificity of systemic inflammatory response syndrome as one of the criteria for sepsis [1-3]. According to the Third International Consensus Definition for Sepsis and Septic Shock (Sepsis-3) in 2016, sepsis was defined as a life-threatening organ dysfunction resulting from dysregulated host responses to infection, with the Sequential Organ Failure Assessment (SOFA) score evaluating the degree of organ dysfunction [4, 5]. The definition of sepsis in Sepsis-3 is similar to the previous definition of severe sepsis in Sepsis-2. A meta-analysis incorporating 27 studies from seven high-income countries showed that the incidence rate and case fatality ratio of severe sepsis were 270 per 100000 person-years and 26\%, respectively (Sepsis-2 definition) [6]. The latest data from the 2017 Global Burden of Diseases, Injuries and Risk Factor Study

Provenance: Submitted article peer-reviewed.

Received: 06 Feb 2020 | Accepted after revision: 04 April 2020

Copyright CERS 2020. This article is open access and distributed under the terms of the Creative Commons Attribution Non-Commercial Licence 4.0. 
showed that 48.9 million incident cases of sepsis and 11.0 million sepsis-related deaths were reported globally in 2017, with the highest age-standardised incidence and mortality of sepsis occurring in areas with the lowest socio-demographic index [7]. The global burden of sepsis is larger than previously estimated and may continue to be great because of a prolonged life expectancy and an ageing population [8-11]. In 2017, the World Health Organization and World Health Assembly recognised sepsis as a global health priority, and adopted a resolution to improve the prevention, diagnosis and management of sepsis [12].

Bacteria have been shown to be the predominant pathogens of sepsis caused by infection [9]. The reported proportions of gram-positive and gram-negative organisms among adult septic patients were both around $40 \%$, while the reported proportions of viruses were very low $[13,14]$. However, the proportion of negative cultures was up to $42 \%$ among patient with sepsis, for whom the possible cause could be virus [15]. Recent studies showed that respiratory viral infections were underdiagnosed in patients with sepsis or septic shock $[16,17]$. In both these studies, conducted in three middle-income countries from Southeast Asia and in a rural area of a high-income country (Sweden), viruses were detected in around one-third of adult patients with sepsis. The viruses, which can cause severe disease, included influenza A and B, respiratory syncytial virus, coronavirus, human metapneumovirus, parainfluenza virus types 1-3, adenovirus, enteroviruses, and rhinovirus [18-20]. Our CAP-China study, which was conducted in 34 hospitals from 10 provinces of mainland China, showed that the proportions of patients with community-acquired pneumonia (CAP) who developed sepsis during hospitals were 40.1 and $39.6 \%$ among those with influenza and non-influenza viral infections, respectively [18]. As well as for commonly detected viruses, emerging novel virus infections can also result in sepsis and have raised global health concerns [20], these include: severe acute respiratory syndrome-coronavirus (SARS-CoV) [21]; Middle East Respiratory Syndrome-coronavirus (MERS-CoV) [22]; and SARS-CoV-2 which caused the recent outbreak of the coronavirus disease 2019 (COVID-19) in China and in many other countries all over the world.

According to the Surviving Sepsis Campaign, intravenous antibiotics within $1 \mathrm{~h}$ after recognition of both sepsis and septic shock is strongly recommended [23]. This recommendation was based on previous findings that a delay in first antibiotic administration was associated with an increased in-hospital mortality [24, 25]. Apart from the benefit of empirical antibiotic use for patients with sepsis, a more precise prescription of antimicrobial therapy, including antiviral therapy for patients without bacterial infection, should be further explored [26]. It is urgent to pay more attention to the role a virus plays in sepsis. The most common sites of infection among patients with sepsis are the respiratory tract (64-68\%), followed by the abdominal tract, bloodstream, and renal and urinary tract $[14,15,27]$. In this review, we mainly focus on respiratory viral infection that could result in sepsis. Viral sepsis has been defined as life-threatening organ dysfunction due to a dysregulated host response to viral infection [28].

\section{Epidemiology of respiratory viral sepsis}

Pneumonia was found to be the most common cause of sepsis and septic shock [14, 29]. A recent retrospective cohort study that included hospitalised patients diagnosed as viral CAP without bacterial co-infection showed viral sepsis was present in $61 \%$ of these patients [30]. According to previously published data, 100 million cases of viral CAP occur every year in adults globally [31], so we can speculate the disease burden of viral sepsis is huge. The most common virus detected among patient with viral sepsis was influenza A virus, followed by rhinovirus, parainfluenza virus types 1-3, respiratory syncytial virus, adenovirus, influenza B virus and coronavirus [30]. A positive pathogen result for a virus is not sufficient for the diagnosis of viral sepsis. Whether the virus detected caused sepsis or not, such as being a coinfection of an unknown pathogen, leading to secondary infection of the other pathogen, or being just a false-positive result, needs to be decided by physicians according to clinical features and both laboratory and radiographic results of the patient [28, 32, 33].

\section{Influenza virus related sepsis}

Influenza viruses, including influenza $\mathrm{A}$ and $\mathrm{B}$, can cause both seasonal epidemics and out-of-season sporadic cases and outbreaks [34]. The annual attack rate of influenza was estimated to be around $10 \%$ among adults $[34,35]$. Most people have self-limited upper respiratory tract symptoms, while some people develop severe illness. Seasonal influenza epidemics were estimated to account for about 291243-645832 respiratory deaths annually, with the highest mortality rate in sub-Saharan Africa and southeast Asia [36]. A retrospective cohort study using hospitalisation data and influenza surveillance data from the USA found that the incidence rate of influenza-associated severe-sepsis hospitalisation was 8.8 per 100000 person-years (95\% CI 3.9-16.5) [37]. Severe sepsis was present in $73 \%$ of influenza-associated critical illness hospitalisations defined as any hospitalisations with acute respiratory failure, severe sepsis, or in-hospital death.

A recent study showed that the most commonly detected virus among viral CAP patients who developed viral sepsis without bacterial co-infection was the influenza A virus, with a detection rate around seven 
times that of influenza B (52\% versus 7\%) [30]. This may be due to different virulences of different strains of influenza virus and the specific host response to them [38, 39]. Previous studies also provide epidemiological data for sepsis related to different subtypes of influenza A virus [40-43]. Data from 26 patients infected with influenza A (H7N9) virus from one province in China showed that 10 (38.5\%) developed septic shock quickly after the onset of illness, which was independently associated with mortality after multivariable adjustment [40]. For hospitalised patients infected with the 2009 pandemic influenza $\mathrm{A}(\mathrm{H} 1 \mathrm{~N} 1)$ (H1N1pdm09), the relative risk of sepsis and septic shock was 1.70 (95\% CI $1.46-$ 1.97) compared with hospitalised patients infected with seasonal influenza [41]. Furthermore, one study conducted among hospitalised patients with H1N1pdm09 showed that the proportion of sepsis was higher among patients with pneumonia than those without (18\% versus 3\%) [42]. The study results listed above not only provide epidemiological characteristics of influenza but also highlight that patients diagnosed with pneumonia during the pandemic season of influenza should be paid more attention by physicians because they are more likely to develop sepsis.

\section{Coronavirus-related sepsis}

The emerging novel coronavirus outbreak in China and many other countries worldwide has brought coronavirus back into our vision. To date, seven types of coronavirus are known to cause human disease, with four of them causing mild infections, while the other three betacoronaviruses, including SARS-CoV, MERS-CoV, and the recently isolated SARS-CoV-2, cause fatal cases [20, 44, 45]. SARS-CoV and MERS-CoV have caused 10590 cases together, with 1632 being fatal cases. As of 10 June 2020, 84652 cases of SARS-CoV-2 infection were confirmed in China, of which 4645 were fatal [46]. Another 1797 imported cases from overseas were reported. In other countries, territories or areas outside China, as of 10 June 2020, more than seven million COVID-19 cases were also confirmed, of which 403380 were fatal [47].

Drosten et al. [22] reported that a 73-year-old man infected with MERS-CoV developed renal insufficiency and required dialysis on day 14 after onset of symptoms. With haemolysis and acute coagulation disorder, this patient died on day 18 due to septic shock. Infections with SARS-CoV were also reported to result in sepsis [21]. According to data from the first 41 cases infected with SARS-CoV-2, platelet counts were decreased while bilirubin and creatinine were elevated in several patients, which were signs of coagulation disorder, liver and renal dysfunction, respectively [20]. These patients can be diagnosed as having sepsis with the updated Sepsis-3 definition. Our recent study included 191 laboratory-confirmed COVID-19 patients in Wuhan who were discharged or died as of 31 January 2020. No bacterial pathogen was detected among these patients on admission. The results showed that the proportions of patients with sepsis and septic shock were $59 \%$ and $20 \%$, respectively. All patients who died and $42 \%$ of patients who were discharged developed viral sepsis during hospitalisation. The median (interquartile) time from illness onset to sepsis was 9.0 (7.0-13.0) days [48].

\section{Other respiratory virus-related sepsis}

Among susceptible populations with sepsis, almost any virus could be detected, including respiratory viruses (e.g. rhinovirus, parainfluenza virus types $1-3$, respiratory syncytial virus, adenovirus, coronavirus and cytomegalovirus) and other viruses (e.g. dengue viruses, hantaviruses, rotavirus and bocavirus) [17, 22, 30, 48-55]. We mainly focus on respiratory viruses related to sepsis. Our study results from CAP-China found the risk of sepsis during hospitalisation between CAP patients with influenza and those with non-influenza respiratory virus infection were not statistically different (OR 1.00 (95\% CI) 0.63-1.58) [18]. Previous studies showed diverse positive rates of non-influenza virus for patients with different clinical characteristics. Among viral CAP patients without bacterial co-infection and severe immunosuppression who developed viral sepsis, the most frequently detected non-influenza respiratory virus was rhinovirus (14\%), followed by parainfluenza virus types $1-3$ (11\%), respiratory syncytial virus (10\%), adenovirus (8\%), and coronavirus (1\%) [30]. Cytomegalovirus is one of the most common viral pathogens detected in immunocompromised patients. In the study conducted among Ugandan adults with sepsis of whom $84 \%$ were infected with HIV, the most common respiratory virus detected was cytomegalovirus, with a positive rate of $41 \%$ [56]. After multivariable adjustment, cytomegalovirus was associated with in-hospital mortality (OR 3.2; 95\% CI, 2.1-10.0). A previous meta-analysis that included studies conducted among immunocompetent patients also suggested that cytomegalovirus was more likely to be detected among patients with severe sepsis or septic shock than mixed patients with or without severe sepsis (32\% versus 15\%) [57]. These results indicate that the specific pathogen spectrum among different populations may be attributable to, but not limited to, the clinical characteristics of study populations, such as immune status.

The aetiological and causal relevance of both influenza and non-influenza respiratory viruses with sepsis is still challenging and needs to be further identified among other prospective cohort studies. Studies conducted in both immunocompetent and immunocompromised hosts that can characterise host responses to viral infections are also needed to better determine causality. 


\section{Pathophysiology of respiratory viral sepsis}

Respiratory viral sepsis is a highly heterogeneous and multifaceted syndrome characterised by an overwhelming and systemic dysregulated host immune response to respiratory viral infection, with organ dysfunction including, but not limited to, the lung. Previous studies provided evidence for extrapulmonary organ dysfunction caused by respiratory viral infection: e.g. acute kidney injury and cardiac injury among cases with influenza infection; acute kidney injury and thrombocytopenia reported for MERS-CoV infection; high viral loads in the gut and liver and moderate viral loads in the kidney among fatal cases with SARS-CoV infection; and liver dysfunction reported for respiratory syncytial virus infection [58-62]. The recent findings from our study showed that around half of the COVID-19 fatalities developed acute kidney injury, heart failure or coagulopathy [48]. In another recent study that included 183 COVID-19 patients, disseminated intravascular coagulation was observed in $71.4 \%$ of fatal cases, and in $0.6 \%$ of non-fatal cases [63]. The multi-organ dysfunction determines that viral sepsis is a more complicated clinical status than severe viral pneumonia, with inflammation in the lung which is the primary and specific target organ of the respiratory virus. The type of infection and host response to the specific pathogen are determinants of sepsis and closely related to prognosis after the development of sepsis. The pathophysiology of sepsis includes that the immune response initiated by an invading pathogen fails to return to homeostasis, and thus culminating in a pathological syndrome that is characterised by sustained excessive inflammation and immune suppression [64].

The initial sensing of the host innate system after infection is to recognise pathogen-associated molecular patterns mediated by innate pattern recognition receptors (PRRs), including Toll-like receptors (TLRs), retinoic acid-inducible gene-1 like receptors, NOD-like receptors, and C-type lectin receptors [65, 66]. For most of the infections, the host innate immune system can eliminate the pathogen through pro-inflammatory responses, including the release of cytokines and chemokines (tumour necrosis factor (TNF), interleukin (IL)-1 $\beta$, IL-12 and IL-18), the recruitment of phagocytes, and the local activation of the complement and coagulation systems [64, 66, 67]. Among patients with sepsis, pathogens cannot be eliminated by the host immune system and the homeostasis of the host immune system is disturbed, resulting in both an excessive inflammation and immune suppression.

The excessive inflammation of sepsis is mediated through the release of pro-inflammatory mediators by leukocytes and parenchymal cells, endothelium and platelets [68-71]. Leukocyte and parenchymal cell injury results in the release of damage-associated molecular patterns, further disrupting the host response by activating many of the PRRs [72, 73]. These PRRs can also recognise pathogen-associated molecular patterns, leading to a vicious cycle that also involves organ damage and dysfunction. The coagulation system, complement system, neutrophils and vascular endothelium are also activated in this stage [74-77]. In the immune suppression stage, both adaptive and innate immune systems are involved. This stage is characterised by the apoptosis of T-cells, B-cells and dendritic cells, the exhaustion of T-cells, and the expansion of regulatory T-cell and myeloid-derived suppressor cell populations [78-80]. Patients with sepsis have increased numbers of myeloid-derived suppressor cells, which are immature myeloid cells that can impede immune responses, particularly T-cell function. Reprogramming of antigen-presenting cells leads to a reduced HLA-DR expression and a diminished capacity to produce pro-inflammatory cytokines [81]. The most important findings in sepsis are the delayed apoptosis of neutrophils and the appearance of immature band-like neutrophils in peripheral blood that have deficits in antimicrobial effector functions [64].

\section{Host immune response to respiratory virus}

The causes and characteristics of sepsis can be highly heterogeneous [64]. However, few studies provide evidence as to whether the pathophysiology of viral sepsis is different from that of bacterial sepsis, mainly because of the limited studies conducted that focus on viral sepsis. Current knowledge of the pathophysiology of respiratory viral sepsis is limited to the specific immune responses to viral infection.

For the influenza virus, haemagglutinins of different strains determine attachment to the epithelial of which specific part of the airway, and the viral polymerase complex is associated with different levels of viral replication and cytokine production in the infected epithelial cells [39, 82, 83]. Seasonal influenza, such as $\mathrm{H} 3 \mathrm{~N} 2$ and $\mathrm{H} 1 \mathrm{~N} 1$, targets preferential epithelium in the large airways (trachea, bronchi and bronchioles) by binding to $\alpha 2,6$-sialylated glycans, while $\mathrm{H} 1 \mathrm{~N} 1 \mathrm{pdm} 09$ and $\mathrm{H} 5 \mathrm{~N} 1$ tend to infect both large airways and alveoli by binding to $\alpha 2,3$-sialylated glycans of pneumocytes [84-88]. Furthermore, mutation in the haemagglutinins of influenza leads to alteration of the cell tropism. The mutation of haemagglutinins of $\mathrm{H} 5 \mathrm{~N} 1$ results in the ability to bind not only $\alpha 2,3$ - but also $\alpha 2,6$-sialylated glycans of pneumocytes $[39,88,89]$. Mutation in the viral RNA polymerase complex could result in better viral replication or increased secretion of pro-inflammatory cytokines [39].

Viral infections, such as the influenza virus, can also trigger initial sensing of the host innate system and recruitment of leukocytes through PRRs [90]. These PRRs include TLR-3, TLR-4, TLR-7, and RIG-I, the 
polymorphisms of which are associated with the susceptibility and severity of influenza virus infection in different individuals [91-96]. Different to bacteria, an influenza virus invades the alveolar epithelial cells first but not alveolar endothelial cells $[82,97]$. Pro-inflammatory cytokines, such as TNF- $\alpha$, IL-1 $\beta$, IL-6 and IL- 8 are produced by the infected epithelial cells and can damage the epithelial-endothelial barrier [82]. Endothelial cells can also be damaged through remodelling of the cellular cytoskeleton, loss of intercellular junctional integrity and cellular apoptosis. These processes lead to pulmonary oedema and respiratory insufficiency, which could further develop into severe pneumonia, acute respiratory distress syndrome and sepsis [98].

Data from the first 41 cases with confirmed SARS-CoV-2 infection showed that cytokines and chemokines, including IL-1 $\beta$ and TNF $\alpha$, were higher in both intensive care unit (ICU) patients and non-ICU patients than healthy controls [20]. These indicators suggest that the host innate immune system initiated eliminating SARS-CoV-2 through a pro-inflammatory response, which is the same as the early stages of sepsis. Furthermore, a higher level of IL-10 was also observed among infected patients compared with healthy controls. The increased secretion of T-helper- 2 cytokines that suppress inflammation suggested that immune suppression may present at an early stage after infection of SARS-CoV-2, which is different from sepsis as the immune-suppressive phase often presents after a pro-inflammatory and excessive inflammatory response. Recent pathological findings show that viral inclusions were found in some alveolar epithelial cells and macrophages of COVID-19 patients [99]. Inflammatory infiltrates dominated by monocytes and macrophages were seen in the lungs. The counts of CD4 and CD8 T-cells were substantially reduced in the spleen and lymphonodus. Furthermore, hepatic stestosis was observed in liver, and interstitial monocyte and lymphocyte inflammatory infiltrates were observed in the heart tissue. These findings need to be further investigated as more biopsy specimens become available.

Viral reactivation may also play a role in the prognosis of sepsis. Immune exhaustion during sepsis provides the probability for some latent infections to escape immunological control, and replicate under this more forgiving environment [28]. Some reactivated viral infections, such as the Epstein-Barr virus, were reported to be associated with clinical outcomes among patients with sepsis $[28,100]$. The potential mechanism is that reactivated viral microRNAs might be involved in sepsis by functional mimicry mechanisms with cellular microRNAs produced by the human genome, sharing the regulation of the same signalling pathways and regulating the same spectrum of microRNAs. For cytomegalovirus, the proportion of its reactivation in immunocompetent patients was around $30 \%$ and has become an area of increasing interest in recent years [101]. Previous studies provided the possibility for the causal relationship between cytomegalovirus reactivation and clinical outcomes among immunocompetent patients with sepsis [102]. However, definite evidence and a mechanism for this relationship are still not clear, e.g. no significant association between cytomegalovirus reactivation and host response biomarkers, including IL-6, IL-10, interferon-gamma-induced protein-10 or IL-1 receptor antagonist, was found among patients with sepsis [103]. Whether cytomegalovirus reactivation plays a role for disease progression or is only a marker of immune suppression in patients with sepsis needs to be further demonstrated.

\section{Interaction of bacterial and viral infection for sepsis}

As mentioned previously, immune suppression characterised by a decreased function in both innate and adaptive immunity following excessive inflammation in almost all forms of sepsis gives way to viral reactivation, which refers to the process of a latent virus switching to a lytic phase of replication. Immune suppression can also increase the probability for secondary bacterial infections, which is associated with increased mortality $[38,64]$. Previous studies showed the interaction between viral and bacterial infection. The interaction of influenza with Streptococcus pneumoniae and Staphylococcus aureus (including methicillin-resistant $S$. aureus) was observed and can be reflected by an increased bacterial coinfection during seasonal epidemic and pandemic outbreaks of influenza. A previous study showed that bacterial coinfection was identified in approximately one-third of fatal H1N1pdm09 cases, with S. pneumoniae identified in $45.5 \%$ of cases and $S$. aureus in $31.8 \%$ of cases (including $71.4 \%$ of the $S$. aureus as methicillin-resistant S. aureus ) with bacterial coinfection [104]. Not only for influenza, respiratory syncytial virus was also reported to increases the virulence of $S$. pneumoniae [105]. Several mechanisms can provide clues to the predisposition of bacterial infection after respiratory viral infections. Respiratory viruses can damage the respiratory epithelium and the basement membrane of the epithelium is exposed, to which bacteria can adhere [106]. Platelet-activating factor receptor upregulated by released pro-inflammatory cytokines can provide a receptor for pneumococcal adherence and invasion [106, 107]. In addition, antibacterial defence mechanisms can be impaired by influenza through increasing neutrophil apoptosis, and neutrophil and monocyte dysfunction $[108,109]$. This epidemiological evidence and the possible mechanisms provide indirect or direct evidence for bacterial reactivation at the stage of immune suppression among patients with viral sepsis. 


\section{Diagnosis of respiratory viral sepsis}

The diagnosis of respiratory viral sepsis depends on two steps: one step is the diagnosis of sepsis using the SOFA score, and the other important and challenging step is identifying the cause of the sepsis as a respiratory virus. The differentiation between bacterial and viral sepsis, especially at the initial stage after the development of sepsis, is important for the treatment of sepsis and prevention of mortality from sepsis. However, no golden standard was identified to broadly and efficiently determine and differentiate the presence and type of infection.

Pathogen detection is the most important step of differential diagnosis between respiratory viral and bacterial sepsis. Point-of-care testing and next-generation sequencing provide the possibility for a quick and accurate identification of the potential pathogen that is causing the sepsis. Next-generation sequencing is especially important for confirmation of infection by novel viruses. The role next-generation sequencing played in the laboratory confirmation of SARS-CoV-2 infection is important. Testing multiple pathogens in one test and saving time are the advantages of point-of-care testing, which are especially important for sepsis [110]. Furthermore, the use of point-of-care testing for sepsis was not limited to pathogen detection, but was also used for blood plasma protein quantification (e.g. C-reactive protein and procalcitonin) and leukocyte monitoring (through antibody capture or intrinsic property characterisation) [111]. Clinical characteristics, blood biomarkers including C-reactive protein and procalcitonin, were not fully demonstrated to clearly discern viral and bacterial infection among patients with pneumonia, while the discrimination ability among patients with sepsis needs to be further demonstrated [112].

To distinguish infection compared to inflammation in the absence of infection and viral infection as compared to bacterial infection, several transcriptomics studies have been conducted to determine the presence of infection as compared to inflammation without infection [113], as well as to distinguish between the presence of bacterial and/or viral infection [114-117]. Some of these studies derived and validated models focusing on gene sets that can distinguish between viral and bacterial infection [117, 118]. However, the many genes required for these models enlarged the difficulty to translate them into practical clinical tools. Sweeney and co-workers $[113,114]$ derived and validated the "Sepsis MetaScore" based on 11 differentially expressed genes and the "Bacterial/viral MetaScore" based on seven differentially expressed genes which can profile the host gene response to build an integrated antibiotics decision model for sepsis. The sensitivities of this model for detecting bacterial infection and the specificity for viral infection were high $(94.0 \%$ and $90.6 \%$, respectively), but the specificity of this model for detecting bacterial infection and sensitivity for viral infection were not satisfying (59.8\% and 53.0\%, respectively). These results show the possibility to quickly discriminate between viral and bacterial sepsis and inform future research to identify biomarkers that can be translated to the clinical setting. Stratifying sepsis patients into more homogeneous subgroups should be the key points for future biomarker research, which can be realised with more consideration for pathophysiology of biomarkers.

\section{Treatment of respiratory viral sepsis}

Timely intervention is the key to effective treatment among patients with sepsis. These include an initial fluid resuscitation and antibiotic therapy within the first hour [119-121]. In patients with haemodynamic instability after the initial fluid resuscitation, further haemodynamic stabilisation and assessment of fluid responsiveness should be continued $[119,122,123]$. During the disease progression of COVID-19, some patients with viral sepsis have clinical features including cold extremities, weak peripheral pulses and severe metabolic acidosis, while the blood pressure levels remain normal. These clinical features indicate the continuing internal environmental disorders and microcirculation dysfunction among these patients. Thus, haemodynamic stabilisation is necessary and important throughout the progress of treatment for patients with viral sepsis. The recommendation of antibiotic therapy is for all patients with sepsis. As mentioned above, a previous study showed that the proportion of sepsis cases with a negative culture was around $42 \%$ [15]. Future studies to evaluate effectiveness of antibiotic use and potential antibiotic resistance among these patients are needed, as inappropriate prescription can increase antibiotic resistance.

Pathogen-directed therapy should be the emphasis during treatment for patients with sepsis. For patients with suspected or confirmed respiratory viral sepsis, the early initiation of antiviral drugs with inhibiting viral replication and decreasing viral load is the most important step. Around 90 antiviral drugs have been formally approved for the treatment of human infectious diseases over the past 50 years, covering viruses that could cause viral sepsis, such as the influenza virus, human cytomegalovirus and respiratory syncytial virus [124]. However, studies with these antiviral drugs were rarely conducted to evaluate the effectiveness for respiratory viral sepsis, which should be the focus of future research. Current findings indicate the potential effect of baloxavir, oseltamivir, peramivir and zanamivir for influenza infections and cidofovir for adenovirus infections in immunocompromised patients [125]. Furthermore, the broad-spectrum antiviral 
drug ribavirin for the treatment of immunosuppressed patients with rhinovirus and respiratory syncytial virus infections, and arbidol for rhinovirus, respiratory syncytial virus, adenovirus and parainfluenza virus infections were also suggested. The broad-spectrum antiviral drugs, which refer to antivirals targeting viral entry and replication or modulating cellular defence systems, should be distinguished from broad-spectrum antibiotics which act against both gram-positive and gram-negative bacteria. To our knowledge, the potential effectiveness of ribavirin for rhinovirus infection and cidofovir for adenovirus infection were only indicated by several case reports, and need to be further demonstrated. Faced with the great challenge brought by SARS-CoV-2, our research group initiated two randomised controlled trials to evaluate the effectiveness and safety of remdesivir among COVID-19 patients, with one conducted among severe patients (clinicaltrials.gov identifier NCT04257656) and the other one among mild and moderate patients (clinicaltrials.gov identifier NCT04252664) [126, 127]. Another trial evaluating the combined use of lopinavir/ritonavir in patients with SARS-CoV-2 infection has been completed (ChiCTR identifier ChiCTR2000029308). Looking back at the history of this infectious disease outbreak, antiviral treatment is the most important and powerful weapon to fight against the emerging and re-emerging viral pathogen, which should be a continuous focus of future research.

The potentially beneficial effects from the early initiation of antiviral treatment and optimal duration of antiviral drugs use among septic patients are not clear, with current study findings limited to severe or critical patients with virus infection. Findings from ICU patients with the H1N1pdm09 virus infection showed the initiation of antiviral treatment within $6 \mathrm{~h}$ of admission was associated with shorter lengths of hospital stay [128]. This indicates that antiviral treatment for critically ill patients with suspected pandemic influenza virus infection should be initiated as early as possible without waiting for the pathogen results. The duration of antiviral drug use is also not yet ascertained for the prolonged viral shedding among patients with critical illness [129]. Previous studies recommended the usage of antiviral drugs for at least 5 days and to repeat pathogen testing among patients at high risk of severe and life-threatening disease [130]. Our future understanding of viral sepsis and the effect of the early use of antiviral medicines will provide evidence to guide the use of antiviral drugs among patients with sepsis. As well as the potential role of antiviral drugs for viruses that causes sepsis, they were indicated in some studies to play a role in the treatment of viral reactivation, which can occur in the stage of host immune suppression. Future studies are still needed to evaluate the treatment effects of antiviral drugs for viral reactivation, with potential applications for all types of sepsis [131].

In recent years, an area of great interest to clinicians and the research of sepsis is immunomodulatory therapy for treating the host immune response. Excessive inflammatory responses can be inhibited by immunomodulatory therapy through altering or counteracting host inflammatory mediators, such as TNF and IL-1, or using broad-spectrum anti-inflammatory molecules with non-selective suppression of inflammation $[132,133]$. However, previous clinical trials to assess the effect of the inhibition of the excessive inflammatory response of septic patients did not show an improvement in the outcome [134, 135]. Several clinical trials to evaluate the effect of anti-inflammatory agents, such as humanised C5a-specific monoclonal antibody and soluble recombinant human thrombomodulin, are still ongoing and cannot provide available data now $[136,137]$. Due to the failure to generate new treatments, immunomodulatory therapy with inhibition of excessive inflammatory response has become less popular, whereas immune stimulants have been advocated to be given to patients with sepsis for the potential effect to reverse immune suppression among sepsis cases [138]. The immune stimulatory system is intended to restore immune functions and promote the rapid clearance of pathogens, and thus reduce the incidence of secondary infections and late sepsis mortality. The use of some immune-stimulating cytokines, such as type II interferon- $\gamma$, IL-7, IL-15 and granulocyte-macrophage colony-stimulating factor, has been demonstrated to reverse immunosuppression in animal models or clinical trials [139, 140]. As the immune stimulatory aims to restore immune function and reduce mortality related to secondary infections of sepsis, the application of this treatment should be specific to patients who may benefit from this therapy. The biomarkers, such as the reduced expression of monocyte HLA-DR and increased expression of monocyte programmed death ligand-1, may be considered for selecting patients who can benefit from immune stimulation [79, 141]. Current knowledge of the potential benefit of immune stimulants therapy for sepsis provides clues for the generation of new treatments in the future. However, due to the limited studies of sepsis treatment and the limited attention from researchers and physicians about viral sepsis, there is a long way to go to evaluate the potential use of these relatively novel therapies from bench to bedside. The subtypes of sepsis also need to be fully considered in the studies conducted in the future.

For other therapies, which include glycaemic control and nutritional support for the treatment of sepsis, controversies also exist. The current consensus for the control of glycaemia is to maintain the glycaemic level at $<180 \mathrm{mg} \cdot \mathrm{dL}^{-1}[23]$, but to avoid tight glycaemic control because of the potential harm from 
hypoglycaemic episodes [142]. Although optimal nutritional support is important in critically ill patients, the timing, dose, duration and route of nutritional support are not clear. Previous studies did not find a superiority of the enteral compared with the parenteral route for association with mortality, but found a greater risk of digestive complications among those who received enteral nutrition [143, 144]. Animal studies showed that anorexia was protective but nutritional supplementation was detrimental in bacterial sepsis, with glucose necessary and sufficient for these effects [145]. In contrast, nutritional supplementation protected against mortality from influenza infection and viral sepsis, while blocking glucose utilisation was lethal. Whether these results can be translated to a clinical setting needs to be further validated among patients with viral or bacterial sepsis.

\section{Conclusions}

Sepsis is a heterogeneous syndrome identified as a life-threatening organ dysfunction that results from dysregulated host responses to infection. As pathogens of sepsis, viruses have not been received enough attention by physicians and researchers, which should be altered given the huge burden. Although our current understanding of the pathophysiology of sepsis has improved, the development of new treatments for sepsis seems not to be consistent with the understanding of the pathophysiology. Future studies should not only focus on understanding the host immune response in the development of sepsis, especially viral sepsis, but also explore how to stratify patients into more homogeneous subgroups on the basis of their pathophysiology. The identification of biomarkers that can differentiate who might benefit from a specific intervention can help the application of new treatments into clinics with monitoring the effects of future therapies. The development of antiviral drugs, immunomodulatory and immune stimulants therapy, and vaccine development, given the important role influenza vaccines play, should be the most important perspectives of future research to fight against the epidemic, emerging and re-emerging viral pathogens that can cause respiratory viral sepsis. Furthermore, with the growing knowledge of sepsis and more patients surviving sepsis in the future, the long-term sequelae of sepsis will be another problem for physicians and researchers to consider.

Conflict of interest: None declared.

Support statement: This work is funded by the Special Project for Emergency of the Ministry of Science and Technology (2020YFC0841300), Chinese Academy of Medical Sciences (CAMS) Innovation Fund for Medical Sciences (CIFMS 2018-I2M-1-003), National Science Grant for Distinguished Young Scholars (81425001/H0104), the National Key Research and Development Program of China (2018YFC1200102), and The Beijing Science and Technology Project (Z19110700660000). Funding information for this article has been deposited with the Crossref Funder Registry.

\section{References}

1 Levy MM, Fink MP, Marshall JC, et al. 2001 SCCM/ESICM/ACCP/ATS/SIS International Sepsis Definitions Conference. Crit Care Med 2003; 31: 1250-1256.

2 Bone RC, Balk RA, Cerra FB, et al. Definitions for sepsis and organ failure and guidelines for the use of innovative therapies in sepsis. The ACCP/SCCM Consensus Conference Committee. American College of Chest Physicians/Society of Critical Care Medicine. Chest 1992; 101: 1644-1655.

3 Cecconi M, Evans L, Levy M, et al. Sepsis and septic shock. Lancet 2018; 392: 75-87.

4 Singer M, Deutschman CS, Seymour CW, et al. The Third International Consensus Definitions for Sepsis and Septic Shock (Sepsis-3). JAMA 2016; 315: 801-810.

5 Vincent JL, Moreno R, Takala J, et al. The SOFA (Sepsis-related Organ Failure Assessment) score to describe organ dysfunction/failure. On behalf of the Working Group on Sepsis-Related Problems of the European Society of Intensive Care Medicine. Intensive Care Med 1996; 22: 707-710.

6 Fleischmann C, Scherag A, Adhikari NK, et al. Assessment of global incidence and mortality of hospital-treated sepsis. Current estimates and limitations. Am J Respir Crit Care Med 2016; 193: 259-272.

7 Rudd KE, Johnson SC, Agesa KM, et al. Global, regional, and national sepsis incidence and mortality, 19902017: analysis for the Global Burden of Disease Study. Lancet 2020; 395: 200-211.

8 Gaieski DF, Edwards JM, Kallan MJ, et al. Benchmarking the incidence and mortality of severe sepsis in the United States. Crit Care Med 2013; 41: 1167-1174.

9 Martin GS, Mannino DM, Eaton S, et al. The epidemiology of sepsis in the United States from 1979 through 2000. N Engl J Med 2003; 348: 1546-1554.

10 Kaukonen KM, Bailey M, Suzuki S, et al. Mortality related to severe sepsis and septic shock among critically ill patients in Australia and New Zealand, 2000-2012. JAMA 2014; 311: 1308-1316.

11 Hodgin KE, Moss M. The epidemiology of sepsis. Curr Pharm Des 2008; 14: 1833-1839.

12 Reinhart K, Daniels R, Kissoon N, et al. Recognizing sepsis as a global health priority - a WHO resolution. N Engl J Med 2017; 377: 414-417.

13 Zahar JR, Timsit JF, Garrouste-Orgeas M, et al. Outcomes in severe sepsis and patients with septic shock: pathogen species and infection sites are not associated with mortality. Crit Care Med 2011; 39: 1886-1895.

14 Vincent JL, Sakr Y, Sprung CL, et al. Sepsis in European intensive care units: results of the SOAP study. Crit Care Med 2006; 34: 344-353.

15 Phua J, Ngerng W, See K, et al. Characteristics and outcomes of culture-negative versus culture-positive severe sepsis. Crit Care 2013; 17: R202.

16 Ljungstrom LR, Jacobsson G, Claesson BEB, et al. Respiratory viral infections are underdiagnosed in patients with suspected sepsis. Eur J Clin Microbiol Infect Dis 2017; 36: 1767-1776. 
Southeast Asia Infectious Disease Clinical Research Network. Causes and outcomes of sepsis in southeast Asia: a multinational multicentre cross-sectional study. Lancet Glob Health 2017; 5: e157-e167.

Zhou F, Wang Y, Liu Y, et al. Disease severity and clinical outcomes of community acquired pneumonia caused by non-influenza respiratory viruses in adults: a multicenter prospective registry study from CAP-China Network. Eur Respir J 2019; 54: 1802406.

Cilloniz C, Ewig S, Polverino E, et al. Microbial aetiology of community-acquired pneumonia and its relation to severity. Thorax 2011; 66: 340-346.

Huang C, Wang Y, Li X, et al. Clinical features of patients infected with 2019 novel coronavirus in Wuhan, China. Lancet 2020; 395: 497-506.

Chua AP, Lee KH. Procalcitonin in severe acute respiratory syndrome (SARS). J Infect 2004; 48: 303-306.

Drosten C, Seilmaier M, Corman VM, et al. Clinical features and virological analysis of a case of Middle East Respiratory Syndrome coronavirus infection. Lancet Infect Dis 2013; 13: 745-751.

997-1000.

Ferrer R, Martin-Loeches I, Phillips G, et al. Empiric antibiotic treatment reduces mortality in severe sepsis and septic shock from the first hour: results from a guideline-based performance improvement program. Crit Care Med 2014; 42: 1749-1755.

Kumar A, Roberts D, Wood KE, et al. Duration of hypotension before initiation of effective antimicrobial therapy is the critical determinant of survival in human septic shock. Crit Care Med 2006; 34: 1589-1596.

Singer M. Antibiotics for sepsis: does each hour really count, or is it incestuous amplification? Am J Respir Crit Care Med 2017; 196: 800-802.

Vincent JL, Rello J, Marshall J, et al. International study of the prevalence and outcomes of infection in intensive care units. JAMA 2009; 302: 2323-2329.

2018; 9: 02147 .

Walden AP, Clarke GM, McKechnie S, et al. Patients with community acquired pneumonia admitted to European intensive care units: an epidemiological survey of the GenOSept cohort. Crit Care 2014; 18: R58.

Cilloniz C, Dominedo C, Magdaleno D, et al. Pure viral sepsis secondary to community-acquired pneumonia in adults: risk and prognostic factors. J Infect Dis 2019; 220: 1166-1171.

Ruuskanen O, Lahti E, Jennings LC, et al. Viral pneumonia. Lancet 2011; 377: 1264-1275.

Dawood FS, Jain S, Finelli L, et al. Emergence of a novel swine-origin influenza A (H1N1) virus in humans. N Engl J Med 2009; 360: 2605-2615.

Kumar S, Wang L, Fan J, et al. Detection of 11 common viral and bacterial pathogens causing communityacquired pneumonia or sepsis in asymptomatic patients by using a multiplex reverse transcription-PCR assay with manual (enzyme hybridization) or automated (electronic microarray) detection. J Clin Microbiol 2008; 46: 3063-3072.

Vaccines against influenza WHO position paper - November 2012. Wkly Epidemiol Rec 2012; 87: 461-476.

Tokars JI, Olsen SJ, Reed C. Seasonal incidence of symptomatic influenza in the United States. Clin Infect Dis 2018; 66: 1511-1518.

Iuliano $\mathrm{AD}$, Roguski $\mathrm{KM}$, Chang $\mathrm{HH}$, et al. Estimates of global seasonal influenza-associated respiratory mortality: a modelling study. Lancet 2018; 391: 1285-1300.

Ortiz JR, Neuzil KM, Shay DK, et al. The burden of influenza-associated critical illness hospitalizations. Crit Care Med 2014; 42: 2325-2332.

Florescu DF, Kalil AC. The complex link between influenza and severe sepsis. Virulence 2013; 5: 137-142.

Fukuyama S, Kawaoka Y. The pathogenesis of influenza virus infections: the contributions of virus and host factors. Curr Opin Immunol 2011; 23: 481-486.

Yang Y, Guo F, Zhao W, et al. Novel avian-origin influenza A (H7N9) in critically ill patients in China. Crit Care Med 2015; 43: 339-345.

Reed C, Chaves SS, Perez A, et al. Complications among adults hospitalized with influenza: a comparison of seasonal influenza and the 2009 H1N1 pandemic. Clin Infect Dis 2014; 59: 166-174.

Jain S, Benoit SR, Skarbinski J, et al. Influenza-associated pneumonia among hospitalized patients with 2009 pandemic influenza A (H1N1) virus - United States, 2009. Clin Infect Dis 2012; 54: 1221-1229.

Han $\mathrm{K}, \mathrm{Ma} \mathrm{H}$, An X, et al. Early use of glucocorticoids was a risk factor for critical disease and death from pH1N1 infection. Clin Infect Dis 2011; 53: 326-333.

World Health Organization. Summary of probable SARS cases with onset of illness from 1 November 2002 to 31 July 2003. www.who.int/csr/sars/country/table2004_04_21/en/ Date last updated: 31 December 2003; date last accessed: 29 January 2020.

World Health Organization. Middle East respiratory syndrome coronavirus (MERS-CoV). www.who.int/ emergencies/mers-cov/en/ Date last updated: 30 November 2019; date last accessed: 29 January 2020.

China National Health Commission. Update on the outbreak of coronavirus disease 2019 (March 4, 2020). www. nhc.gov.cn/xcs/yqfkdt/202006/d89974801e894128b26f6ecc85481334.shtml Date last updated: 10 June 2020; date last accessed: 11 June 2020.

World Health Organization. Coronavirus disease (COVID-2019) situation reports. www.who.int/emergencies/ diseases/novel-coronavirus-2019/situation-reports Date last updated: 10 June 2020; date last accessed: 11 June 2020.

Zhou F, Yu T, Du R, et al. Clinical course and risk factors for mortality of adult inpatients with COVID-19 in Wuhan, China: a retrospective cohort study. Lancet 2020; 395: 1054-1062.

Modhiran N, Watterson D, Muller DA, et al. Dengue virus NS1 protein activates cells via Toll-like receptor 4 and disrupts endothelial cell monolayer integrity. Sci Transl Med 2015; 7: 304ra142.

Zahariadis G, Jerome KR, Corey L. Herpes simplex virus-associated sepsis in a previously infected immunocompetent adult. Ann Intern Med 2003; 139: 153-154.

Martinez E, de Diego A, Paradis A, et al. Herpes simplex pneumonia in a young immunocompetent man. Eur Respir J 1994; 7: 1185-1188.

Pastere S, Jean-Baptiste S. Intensive care admissions for severe chikungunya virus infection, French Polynesia. Emerging Infect Dis 2018; 24: 794-796. 
Rolle A, Schepers K, Cassadou S, et al. Severe sepsis and septic shock associated with chikungunya virus infection, Guadeloupe, 2014. Emerging Infect Dis 2016; 22: 891-894.

Kadambari S, Harvala H, Simmonds P, et al. Strategies to improve detection and management of human parechovirus infection in young infants. Lancet Infect Dis 2019; 19: e51-e58.

Olijve L, Jennings L, Walls T. Human parechovirus: an increasingly recognized cause of sepsis-like illness in young infants. Clin Microbiol Rev 2018; 31: e00047-17.

Moore CC, Jacob ST, Banura P, et al. Etiology of sepsis in Uganda using a quantitative polymerase chain reaction-based TaqMan Array Card. Clin Infect Dis 2019; 68: 266-272.

Kalil AC, Florescu DF. Prevalence and mortality associated with cytomegalovirus infection in nonimmunosuppressed patients in the intensive care unit. Crit Care Med 2009; 37: 2350-2358.

Arabi YM, Arifi AA, Balkhy HH, et al. Clinical course and outcomes of critically ill patients with Middle East Respiratory Syndrome coronavirus infection. Ann Intern Med 2014; 160: 389-397.

Bakalli I. Liver dysfunction in severe sepsis from respiratory syncytial virus. J Pediatr Intensive Care 2018; 7 110-114.

Farcas GA, Poutanen SM, Mazzulli T, et al. Fatal severe acute respiratory syndrome is associated with multiorgan involvement by coronavirus. J Infect Dis 2005; 191: 193-197.

Nin N, Lorente JA, Soto L, et al. Acute kidney injury in critically ill patients with 2009 influenza A (H1N1) vira pneumonia: an observational study. Intensive Care Med 2011; 37: 768-774.

, Wang Y, Gu X, et al. Association between cardiac injury and mortality in hospitalized patients infected with avian influenza A (H7N9) virus. Crit Care Med 2020; 48: 451-458. with novel coronavirus pneumonia. I Thromb Haemost 2020; 18: 1233-1234.

van der Poll T, van de Veerdonk FL, Scicluna BP, et al. The immunopathology of sepsis and potential therapeutic targets. Nat Rev Immunol 2017; 17: 407-420.

Boyd JH, Russell JA, Fjell CD. The meta-genome of sepsis: host genetics, pathogens and the acute immune response. J Innate Immun 2014; 6: 272-283.

Takeuchi O, Akira S. Pattern recognition receptors and inflammation. Cell 2010; 140: 805-820. 987-995.

Opal SM, van der Poll T. Endothelial barrier dysfunction in septic shock. J Intern Med 2015; 277: 277-293.

Tressel SL, Kaneider NC, Kasuda S, et al. A matrix metalloprotease-PAR1 system regulates vascular integrity, systemic inflammation and death in sepsis. EMBO Mol Med 2011; 3: 370-384.

Claushuis TA, van Vught LA, Scicluna BP, et al. Thrombocytopenia is associated with a dysregulated host response in critically ill sepsis patients. Blood 2016; 127: 3062-3072.

de Stoppelaar SF, van 't Veer C, van der Poll T. The role of platelets in sepsis. Thromb Haemost 2014; 112: 666-677.

Deutschman CS, Tracey KJ. Sepsis: current dogma and new perspectives. Immunity 2014; 40: 463-475.

Chan JK, Roth J, Oppenheim JJ, et al. Alarmins: awaiting a clinical response. J Clin Invest 2012; 122: 2711-2719.

Merle NS, Noe R, Halbwachs-Mecarelli L, et al. Complement system part II: role in immunity. Front Immunol 2015; 6: 257.

Silasi-Mansat R, Zhu H, Popescu NI, et al. Complement inhibition decreases the procoagulant response and confers organ protection in a baboon model of Escherichia coli sepsis. Blood 2010; 116: 1002-1010.

Levi M, van der Poll T. Coagulation and sepsis. Thromb Res 2017; 149: 38-44.

Abraham E, Reinhart K, Opal S, et al. Efficacy and safety of tifacogin (recombinant tissue factor pathway inhibitor) in severe sepsis: a randomized controlled trial. JAMA 2003; 290: 238-247.

JAMA 2011; 306: 2594-2605

Hotchkiss RS, Monneret G, Payen D. Sepsis-induced immunosuppression: from cellular dysfunctions to immunotherapy. Nat Rev Immunol 2013; 13: 862-874.

Hotchkiss RS, Tinsley KW, Swanson PE, et al. Depletion of dendritic cells, but not macrophages, in patients with sepsis. J Immunol 2002; 168: 2493-2500.

Liu TF, Yoza BK, El Gazzar M, et al. NAD+-dependent SIRT1 deacetylase participates in epigenetic reprogramming during endotoxin tolerance. J Biol Chem 2011; 286: 9856-9864.

Short KR, Kroeze E, Fouchier RAM, et al. Pathogenesis of influenza-induced acute respiratory distress syndrome. Lancet Infect Dis 2014; 14: 57-69.

Shinya K, Ebina M, Yamada S, et al. Avian flu: influenza virus receptors in the human airway. Nature 2006; 440: 435-436.

Perez-Padilla R, de la Rosa-Zamboni D, Ponce de Leon S, et al. Pneumonia and respiratory failure from swine-origin influenza A (H1N1) in Mexico. N Engl J Med 2009; 361: 680-689.

Guarner J, Falcon-Escobedo R. Comparison of the pathology caused by H1N1, H5N1, and H3N2 influenza viruses. Arch Med Res 2009; 40: 655-661.

Uiprasertkul M, Kitphati R, Puthavathana P, et al. Apoptosis and pathogenesis of avian influenza A (H5N1) virus in humans. Emerging Infect Dis 2007; 13: 708-712.

Ng WF, To KF. Pathology of human H5N1 infection: new findings. Lancet 2007; 370: 1106-1108.

Liu Y, Childs RA, Matrosovich T, et al. Altered receptor specificity and cell tropism of D222G hemagglutinin mutants isolated from fatal cases of pandemic A(H1N1) 2009 influenza virus. J Virol 2010; 84: 12069-12074.

Yamada S, Suzuki Y, Suzuki T, et al. Haemagglutinin mutations responsible for the binding of H5N1 influenza A viruses to human-type receptors. Nature 2006; 444: 378-382.

Teijaro JR, Walsh KB, Cahalan S, et al. Endothelial cells are central orchestrators of cytokine amplification during influenza virus infection. Cell 2011; 146: 980-991

Kawai T, Akira S. Toll-like receptor and RIG-I-like receptor signaling. Ann N Y Acad Sci 2008; 1143: 1-20.

H1N1/2009 influenza in otherwise healthy children. Virol J 2012; 9: 270. 
Guillot L, Le Goffic R, Bloch S, et al. Involvement of Toll-like receptor 3 in the immune response of lung epithelial cells to double-stranded RNA and influenza A virus. J Biol Chem 2005; 280: 5571-5580.

94 Imai Y, Kuba K, Neely GG, et al. Identification of oxidative stress and Toll-like receptor 4 signaling as a key pathway of acute lung injury. Cell 2008; 133: 235-249.

95 Diebold SS, Kaisho T, Hemmi H, et al. Innate antiviral responses by means of TLR7-mediated recognition of single-stranded RNA. Science 2004; 303: 1529-1531.

96 Pichlmair A, Schulz O, Tan CP, et al. RIG-I-mediated antiviral responses to single-stranded RNA bearing 5'-phosphates. Science 2006; 314: 997-1001.

97 Matthay MA, Zemans RL. The acute respiratory distress syndrome: pathogenesis and treatment. Annu Rev Pathol $2011 ; 6: 147-163$

98 Steinberg BE, Goldenberg NM, Lee WL. Do viral infections mimic bacterial sepsis? The role of microvascular permeability: a review of mechanisms and methods. Antiviral Res 2012; 93: 2-15.

99 China National Health Commission. Notice on the issuance of diagnosis and treatment procedure for pneumonia patients infected with the 2019-nCoV (Version 7). www.nhc.gov.cn/yzygj/s7653p/202003/ 46c9294a7dfe4cef80dc7f5912eb1989.shtml Date last updated: 4 March 2020; date last accessed: 5 March 2020.

100 Giza DE, Fuentes-Mattei E, Bullock MD, et al. Cellular and viral microRNAs in sepsis: mechanisms of action and clinical applications. Cell Death Differ 2016; 23: 1906-1918.

101 Marandu T, Dombek M, Cook CH. Impact of cytomegalovirus load on host response to sepsis. Med Microbio Immunol 2019; 208: 295-303.

102 Heininger A, Haeberle H, Fischer I, et al. Cytomegalovirus reactivation and associated outcome of critically ill patients with severe sepsis. Crit Care 2011; 15: R77.

103 van de Groep K, Nierkens S, Cremer OL, et al. Effect of cytomegalovirus reactivation on the time course of systemic host response biomarkers in previously immunocompetent critically ill patients with sepsis: a matched cohort study. Crit Care 2018; 22: 348.

104 Centers for Disease Control and Prevention. Bacterial coinfections in lung tissue specimens from fatal cases of 2009 pandemic influenza A (H1N1) - United States, May-August 2009. MMWR Morb Mortal Wkly Rep 2009; 58: $1071-1074$.

105 Smith CM, Sandrini S, Datta S, et al. Respiratory syncytial virus increases the virulence of Streptococcus pneumoniae by binding to penicillin binding protein 1a. A new paradigm in respiratory infection. Am J Respir Crit Care Med 2014; 190: 196-207.

106 McCullers JA, Rehg JE. Lethal synergism between influenza virus and Streptococcus pneumoniae: characterization of a mouse model and the role of platelet-activating factor receptor. J Infect Dis 2002; 186: 341-350.

107 Hament JM, Kimpen JL, Fleer A, et al. Respiratory viral infection predisposing for bacterial disease: a concise review. FEMS Immunol Med Microbiol 1999; 26: 189-195.

108 Colamussi ML, White MR, Crouch E, et al. Influenza A virus accelerates neutrophil apoptosis and markedly potentiates apoptotic effects of bacteria. Blood 1999; 93: 2395-2403.

109 Nickerson CL, Jakab GJ. Pulmonary antibacterial defenses during mild and severe influenza virus infection. Infect Immun 1990; 58: 2809-2814.

110 Kozel TR, Burnham-Marusich AR. Point-of-care testing for infectious diseases: past, present, and future. J Clin Microbiol 2017; 55: 2313-2320.

111 Oeschger T, McCloskey D, Kopparthy V, et al. Point of care technologies for sepsis diagnosis and treatment. Lab Chip 2019; 19: 728-737.

112 Gilbert DN. Procalcitonin as a biomarker in respiratory tract infection. Clin Infect Dis 2011; 52: Suppl. 4, S346-S350.

113 Sweeney TE, Shidham A, Wong HR, et al. A comprehensive time-course-based multicohort analysis of sepsis and sterile inflammation reveals a robust diagnostic gene set. Sci Transl Med 2015; 7: 287ra271.

114 Sweeney TE, Wong HR, Khatri P. Robust classification of bacterial and viral infections via integrated host gene expression diagnostics. Sci Transl Med 2016; 8: 346ra391.

115 Zaas AK, Burke T, Chen M, et al. A host-based RT-PCR gene expression signature to identify acute respiratory viral infection. Sci Transl Med 2013; 5: 203ra126.

116 Suarez NM, Bunsow E, Falsey AR, et al. Superiority of transcriptional profiling over procalcitonin for distinguishing bacterial from viral lower respiratory tract infections in hospitalized adults. J Infect Dis 2015; 212: $213-222$.

117 Tsalik EL, Henao R, Nichols M, et al. Host gene expression classifiers diagnose acute respiratory illness etiology. Sci Transl Med 2016; 8: 322ra311.

118 Andres-Terre M, McGuire HM, Pouliot Y, et al. Integrated, multi-cohort analysis identifies conserved transcriptional signatures across multiple respiratory viruses. Immunity 2015; 43: 1199-1211.

119 Rivers E, Nguyen B, Havstad S, et al. Early goal-directed therapy in the treatment of severe sepsis and septic shock. N Engl J Med 2001; 345: 1368-1377.

120 Levy MM, Evans LE, Rhodes A. The Surviving Sepsis Campaign bundle: 2018 update. Crit Care Med 2018; 46: 997-1000

121 Seymour CW, Gesten F, Prescott HC, et al. Time to treatment and mortality during mandated emergency care for sepsis. N Engl J Med 2017; 376: 2235-2244.

122 Marik PE, Monnet X, Teboul JL. Hemodynamic parameters to guide fluid therapy. Ann Intensive Care 2011; 1: 1.

123 Cavallaro F, Sandroni C, Antonelli M. Functional hemodynamic monitoring and dynamic indices of fluid responsiveness. Minerva Anestesiol 2008; 74: 123-135.

124 De Clercq E, Li G. Approved antiviral drugs over the past 50 years. Clin Microbiol Rev 2016; 29 : 695-747.

125 Walter JM, Wunderink RG. Severe respiratory viral infections: new evidence and changing paradigms. Infect Dis Clin North Am 2017; 31: 455-474.

126 ClinicalTrials.gov. Mild/Moderate 2019-nCoV Remdesivir RCT. NCT04252664. https://clinicaltrials.gov/ct2/show/ NCT04252664?term=bin Date last updated: 15 April 2020; date last accessed: 7 February 2020.

127 ClinicalTrials.gov. Severe 2019-nCoV Remdesivir RCT. NCT04257656. https://clinicaltrials.gov/ct2/show/ NCT04257656?term=bin+cao\&draw=2\&rank=4 Date last updated15 April 2020; date last accessed: 7 February 2020 
Alvarez-Lerma F, Marin-Corral J, Vila C, et al. Delay in diagnosis of influenza A (H1N1)pdm09 virus infection in critically ill patients and impact on clinical outcome. Crit Care 2016; 20: 337.

129 To KK, Hung IF, Li IW, et al. Delayed clearance of viral load and marked cytokine activation in severe cases of pandemic H1N1 2009 influenza virus infection. Clin Infect Dis 2010; 50: 850-859.

130 Ison MG, de Jong MD, Gilligan KJ, et al. End points for testing influenza antiviral treatments for patients at high risk of severe and life-threatening disease. J Infect Dis 2010; 201: 1654-1662.

131 Razonable RR. Antiviral drugs for viruses other than human immunodeficiency virus. Mayo Clin Proc 2011; 86: 1009-1026.

132 Annane D, Bellissant E, Bollaert PE, et al. Corticosteroids for treating sepsis. Cochrane Database Syst Rev 2015: Cd002243.

133 Vincent JL, Sun Q, Dubois MJ. Clinical trials of immunomodulatory therapies in severe sepsis and septic shock. Clin Infect Dis 2002; 34: 1084-1093.

134 Marshall JC. Why have clinical trials in sepsis failed? Trends Mol Med 2014; 20: 195-203.

135 Venkatesh B, Finfer S, Cohen J, et al. Adjunctive glucocorticoid therapy in patients with septic shock. N Engl J Med 2018; 378: 797-808.

136 ClinicalTrials.gov. Studying Complement Inhibition in Early, Newly Developing Septic Organ Dysfunction (SCIENS). NCT02246595. https://clinicaltrials.gov/ct2/show/NCT02246595 Date last updated: 25 April 2016; date last accessed: 31 January 2020.

137 -ClinicalTrials.gov. Phase 3 Safety and Efficacy Study of ART-123 in Subjects With Severe Sepsis and Coagulopathy. NCT01598831. https://clinicaltrials.gov/ct2/show/NCT01598831 Date last updated: April 212020 ; date last accessed: 31 January 2020.

138 Leentjens J, Kox M, van der Hoeven JG, et al. Immunotherapy for the adjunctive treatment of sepsis: from immunosuppression to immunostimulation. Time for a paradigm change? Am J Respir Crit Care Med 2013; 187: $1287-1293$.

139 Leentjens J, Kox M, Koch RM, et al. Reversal of immunoparalysis in humans in vivo: a double-blind, placebo-controlled, randomized pilot study. Am J Respir Crit Care Med 2012; 186: 838-845.

140 Kasten KR, Prakash PS, Unsinger J, et al. Interleukin-7 (IL-7) treatment accelerates neutrophil recruitment through gamma delta T-cell IL-17 production in a murine model of sepsis. Infect Immun 2010; 78: 4714-4722.

141 Hotchkiss RS, Colston E, Yende S, et al. Immune checkpoint inhibition in sepsis: a Phase 1b randomized, placebo-controlled, single ascending dose study of antiprogrammed cell death-ligand 1 antibody (BMS-936559). Crit Care Med 2019; 47: 632-642.

142 Finfer S, Chittock DR, Su SY, et al. Intensive versus conventional glucose control in critically ill patients. $N$ Engl $J$ Med 2009; 360: 1283-1297.

143 Reignier J, Boisrame-Helms J, Brisard L, et al. Enteral versus parenteral early nutrition in ventilated adults with shock: a randomised, controlled, multicentre, open-label, parallel-group study (NUTRIREA-2). Lancet 2018; 391: $133-143$.

144 Harvey SE, Parrott F, Harrison DA, et al. A multicentre, randomised controlled trial comparing the clinical effectiveness and cost-effectiveness of early nutritional support via the parenteral versus the enteral route in critically ill patients (CALORIES). Health Technol Assess 2016; 20: 1-144.

145 Wang A, Huen SC, Luan $\mathrm{HH}$, et al. Opposing effects of fasting metabolism on tissue tolerance in bacterial and viral inflammation. Cell 2016; 166: 1512-1525. 